$16^{\text {th }}$ International Conference on

AEROSPACE SCIENCES \& AVIATION TECHNOLOGY,

$\boldsymbol{A S A T}$ - 16 - May 26 - 28, 2015, E-Mail: asat@mtc.edu.eg Military Technical College, Kobry Elkobbah, Cairo, Egypt

Tel : +(202) 24025292 - 24036138, Fax: +(202) 22621908

\title{
Structural Health Monitoring of Composite Laminated Plates Using an Array of PWAS
}

\author{
M. ABD EL-RAHEM MAHMOUD*, M. S. AMIN ${ }^{\dagger}$, and M. K. ZIDAN ${ }^{\ddagger}$
}

\begin{abstract}
The advances in using composite materials in different structural application lead to the need of continuous, robust, and structural health monitoring (SHM) systems. One of the most promising techniques for SHM is electromechanical impedance (EMI) technique which depends mainly on the coupling nature of piezoelectric ceramics. Piezoelectric wafer active sensors (PWAS) can be employed as both a well-controlled actuator and sensor at the same time for diagnostic algorithms based on EMI technique. The study presented in this paper interested in EMI technique application to detect damage in composite laminated plates by applying synchronized system of PWAS array operated remotely by exerting harmonic analysis in desired frequency ranges. Frequency ranges are selected upon modal analysis of the healthy tested structure. Harmonic analysis is carried out for different damage scenarios. Extracted electrical charge spectrum data from each PWAS for the previous scenarios can be processed to plot electrical impedance for each case. The modeling process was carried out using a finite element commercial package, ANSYS v.15.0 in which multiphysics-based modeling can be used for such structure made of laminated composite material. The extracted resultant spectrum, for healthy structure, is used as a datum in which it is related to its damaged counterparts through damage identification indices such as root mean square deviation (RMSD), and damage detection index (DDI). These indices were used as indicators for the changes in the modal parameters and hence, yielded reasonable results for both damage quantification and localization purposes.
\end{abstract}

Keywords: Electromechanical impedance, composite materials, piezoelectric wafer active sensors, structural health monitoring.

\footnotetext{
* Graduate Student, Civil Eng. Department, Military Technical College, Egyptian Armed Forces, Egypt.

$\dagger$ Egyptian Armed Forces, Egypt.

Prof. Dr., Civil Eng. Dept., Faculty of Engineering, Ain Shams University, Cairo, Egypt.
} 


\section{Nomenclature}

$\alpha$ Mass matrix multiplier

$\beta$ Stiffness matrix multiplier

[C] Damping matrix

$\left[C^{v h}\right]$ Element dielectric damping matrix

$\left[\mathrm{c}^{\mathrm{s}}\right.$ ] Piezoelectric material stiffness matrix

$\gamma_{13}, \gamma_{12}, \gamma_{23}$ poisson ratios

\{D\} Electric displacement vector

[d] Piezoelectric material strain matrix

$\{\mathrm{E}\}$ Electric field vector

$\mathrm{E}_{1}, \mathrm{E}_{2}, \mathrm{E}_{3}$ Young's moduli in directions $\mathrm{x}, \mathrm{y}$, and $\mathrm{z}$

[e] Piezoelectric stress matrix

$\left[\varepsilon^{\mathrm{T}}\right]$ Permittivity at fixed stress

$\hat{\varepsilon}_{33}^{T}$ Complex permittivity component in $\mathrm{z}$ direction

$\{\mathrm{F}\}$ Force vector

$\mathrm{G}_{13}, \mathrm{G}_{12}, \mathrm{G}_{23}$ Shear moduli

[K] Stiffness matrix

$\left[\mathrm{K}^{\mathrm{Z}}\right]$ Piezoelectric coupling matrix

$\left[\mathrm{K}^{\mathrm{d}}\right]$ Dielectric conductivity matrix

$\left[K^{v}\right]$ Element electrical conductivity coefficient matrix

$\mathrm{k}$ Wave number

$\{\mathrm{L}\}$ Vector of nodal, surface, and body charges

$l_{\mathrm{a}}$ Actuator length

[M] Mass matrix

$\{\mathrm{S}\}$ Strain vector

$\left[\mathrm{s}^{\mathrm{E}}\right]$ Piezoelectric elasticity matrix at fixed field

$\{T\}$ Stress vector

$\mathrm{t}_{\mathrm{a}}$ Actuator thickness

$\rho$ Density

$\{\mathrm{u}\}$ Vector of nodal displacement

$\{v\}$ Vector of nodal electric potential

$\phi$ Displacement and electric potential phase shift

$\mathrm{w}_{\mathrm{a}}$ Actuator width

$\omega$ Angular frequency

$\psi$ Force and charge phase shift

Y Admittance

$\widehat{Y}_{\mathrm{P}}^{\mathrm{E}}$ Complex young's modulus

$\mathrm{Za}(\omega)$ Actuator mechanical impedance

Zs ( $\omega)$ Structure mechanical impedance

\section{Introduction}

SHM, which facilitate maintenance procedure throughout effective monitoring, is carried out in different engineering field such as (aerospace engineering, maritime engineering, civil engineering, mechanical engineering...etc.). It makes sense to clarify that SHM guaranties reducing response time to catastrophic events, on-going assessment of design, assurance of structure's strength and stability, improving maintenance and management strategies and finally reduction in down time which impact maintenance, repair, or rehabilitation cost as well as safety measurements. Extraordinary development of composite material usage in, almost, all engineering fields, and complexity of such material behavior under stresses were 
strong motivations to incorporate smart materials during the manufacturing process of structural elements. These materials are capable of respond spontaneously to external stimuli exerted on the structure[1], such as piezoelectric materials. Piezoelectricity is simply the ability of certain materials (notably crystals and certain ceramics) to produce an electric potential in response to an applied mechanical stress and, conversely, generate a stress in response to an electric stimulus. The mechanical to electrical conversion is called "direct piezoelectric effect" and the reversible effect is termed "converse piezoelectric effect". Many SHM innovative techniques depend on piezoelectric transducers (actuators, sensors, PWAS, etc.) with very promising results, which facilitate the processes of flaws detection at real time, localization of these flaws, identifying them and finally integrity assessment of the structure even before these flaws propagate to be noticeable with minimal man-effort and highest precision available accuracy, using very simple and cheap permanent-mounted pieces of equipment.

Generally, there are two main categories of SHM using piezoelectric materials, wave-based and vibration-based techniques. Acoustic emission, guided ultrasonic waves, phased arrays ...etc., are examples of wave-based techniques. One of the most promising, prominent, and simple vibration-based techniques is electromechanical impedance. Researchers had a great deal of efforts to model SHM process with EMI technique. Piezoelectric materials have significant electromechanical coupling property so; it can be used to serve as both sensor and actuator, which can be defined as piezoelectric wafer active sensor (PWAS). PWAS can be then used, effectively, in SHM for diagnostic algorithms that require a known, well-controlled excitation. That technique which utilizes this advantage of PWAS is referred to as electromechanical impedance(EMI) technique [2]. EMI technique is a common method used for damage detection in SHM which uses the sensitive capability of PWAS to perform a nondestructive inspection of the monitored structure. The main key of using such technique in SHM is the relation between applied electrical field and induced mechanical strain (and vice versa) introduced by the well-known constitutive equation of piezoelectric materials.

$\left[\begin{array}{l}S \\ D\end{array}\right]=\left[\begin{array}{cc}s^{E} & d \\ d & \varepsilon^{T}\end{array}\right]\left[\begin{array}{l}T \\ E\end{array}\right]$
$\left[\begin{array}{l}T \\ D\end{array}\right]=\left[\begin{array}{cc}c^{S} & -e \\ e & \varepsilon^{T}\end{array}\right]\left[\begin{array}{l}S \\ E\end{array}\right]$

This relation identifies electromechanical impedance $\mathrm{Z}_{\mathrm{a}}$ of PWAS as a function of its mechanical properties with free- free boundary conditions. When PWAS bonded onto the structure, and due to its electromechanical coupling characteristics, the electrical impedance measured at its terminal is affected directly by the mechanical impedance $\mathrm{Z}_{\mathrm{s}}$ of the host structure. This can be used to get a mechanical resonance spectrum for such structure represented in spectrum of peaks and valleys of the real part of EMI spectrum, which can be considered as 'structural fingerprint' of the healthy structure. to monitor any changes in its integrity.

Liang et al [3] established a genuine formula to determine the admittance, which is the inverse of the impedance, for a one dimensional PWAS attached to a host structure as shown in Fig. 1.

$Y(\omega)=j \omega \frac{w_{a} l_{a}}{t_{a}}\left(\hat{\varepsilon}_{33}^{T}-d_{31}{ }^{2} \hat{Y}_{P}^{E}+\frac{Z a(\omega)}{Z a(\omega)+Z s(\omega)} \times d_{31}{ }^{2} \hat{Y}_{P}^{E}\left(\frac{\tan k l_{a}}{k l_{a}}\right)\right)$ 


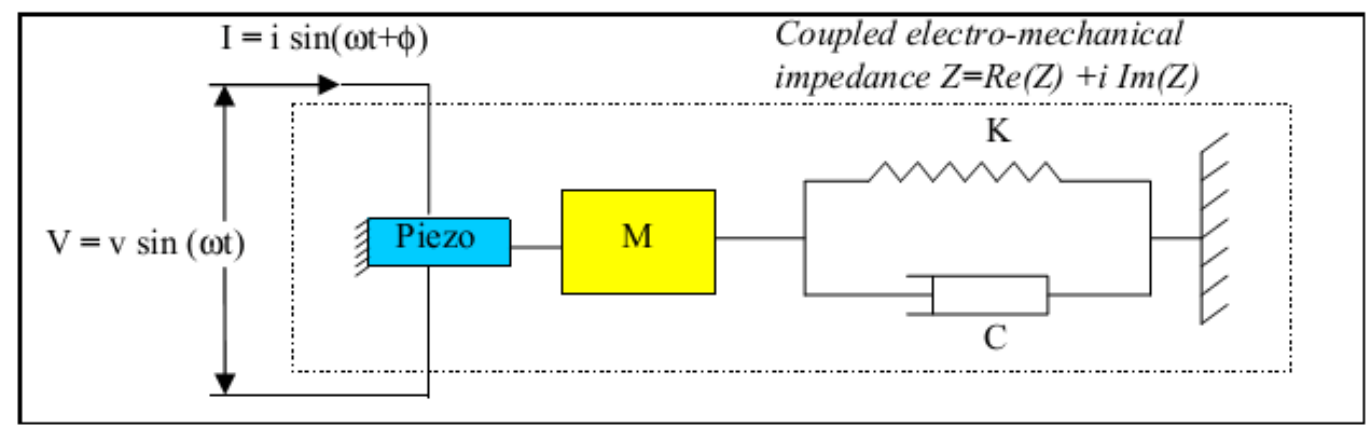

Fig.1. Electromechanical coupling of PWAS and host structure, Liang [3].

This prototype model was improved by many researchers, Zhou et al [4] extended the model to 2-D model while Giurgiutiu and Zagria [5]introduced an approach for admittance of both 1D strip and 2D disc cases , based on derivation of Ikeda[6] for the solutions of classical boundary conditions, and dynamics of the PWAS and dynamics the host structure which is introduced as spring. Bhalla [7] have defined the 'effective impedance', then used a numericalanalytical combined approach to calculate admittance of a square PWAS mounted onto aluminum plate by using FE commercial package to apply harmonic load of unity on the host structure. The calculated mechanical impedance of the host structure at the effective boundary of the PWAS is then included in the 2-D analytical formula to get the admittance signature. Park and Farrar [2] introduced noticeable technique to study self-diagnostic for both PWAS and bonding layer, using the imaginary part of admittance. Gresil et al [8] had a remarkable work in studying SHM using EMI technique for glass fiber reinforced polymer (GFRP) plates for various working conditions including presence of notches and delamination and the effect of damping for several ranges of frequencies. Pierre et al [9] studied usage of multi PWAS for SHM of carbon epoxy composite.

Analytical approaches were results of understanding and analyzing the electrical-mechanical coupling of PWAS as well as the interference mechanism between the mechanical properties of both PWAS and host structure. Nevertheless, these approaches are limited to simple geometry models such as beams and isotropic materials. These limitations impact the reliability and sustainability usage of EMI technique when used in SHM of complex geometries with orthotropic/anisotropic composite materials. The advances developed in FE models correlated with the analytical models such as [10, 11] and the experimental implementations such as [8,9] paved the way to the availability of powerful FE commercial software that can be used as an effective tool to model EMI for complex geometry structure made of anisotropic materials.

In the present research, a numerical simulation study is carried out to investigate the performance of a proposed SHM system for structures made of composite material. The system is composed of an array of four PWAS placed on $250 \times 250 \times 3 \mathrm{~mm}$ composite plate as host structure. Finite element model is constructed using multi-physics-based modeling commercial package, ANSYS v.15.0 in order to simulate EMI technique in SHM of composite plate. The modal parameters for the healthy and simulated damage scenarios for composite plates are identified through numerical simulation of harmonic analysis. The acquired EMI spectrums, in the desired frequency range, for both healthy and its damaged counterparts are used in damage identification procedure. MATLAB codes are developed to implement two damage indices; root mean square deviation (RMSD), and damage detection index (DDI). The results of RMSD and DDI are used as measuring parameters for damage identification. 


\section{Finite Element Model}

The test article is a $(250 \times 250 \times 3 \mathrm{~mm})$ plate made of $2 \mathrm{D}$ Epoxy_Carbon_Woven_395GPa_Preprege. The mechanical properties of the composite plate material are illustrated in Table 1. An advanced element named SOLSH190 is selected from ANSYS library to model the plate as it is used for simulating shell structures with a wide range of thickness (from thin to moderate). The element possesses eight-node connectivity with three degrees of freedom at each node: translations in the nodal $\mathrm{x}, \mathrm{y}$, and $\mathrm{z}$ directions. Thus, connecting SOLSH190 with other continuum elements requires no extra procedures. SOLSH190 can be used for layered applications in which it satisfies the requirements for modeling laminated composite plate of the present study. Fig. 2 illustrates the finite element model of the composite plate and the position of the four PWAS. The boundary conditions for the four plate sides are constrained in the directions of $\mathrm{U}_{\mathrm{x}}, \mathrm{U}_{\mathrm{y}}$, and $\mathrm{U}_{\mathrm{z}}$. Accuracy in modeling composite shells is governed by the first-order shear-deformation theory or Mindlin - Reissner shell theory. An array of four PWAS was arranged symmetrically on the top surface of the plate with the configuration illustrated in Fig.2. Each PWAS is $10 \times 10 \times 0.5 \mathrm{~mm}$ made of APC850 material with characteristics illustrated in Table 1 in accordance with the American Piezoelectric Ceramics APC [12].

Table 1.Mechanical and electrical characteristics of used materials

\begin{tabular}{c|c|c}
\hline \hline Properties & APC850 & $\begin{array}{c}\text { Epoxy_Carbon_Woven_ } \\
\text { 395GPa_Preprege }\end{array}$ \\
\hline$E_{1}, E_{2}$ & $63.24 \mathrm{GPa}$ & $91.82 \mathrm{GPa}$ \\
\hline$E_{3}$ & $54.00 \mathrm{GPa}$ & $9.00 \mathrm{GPa}$ \\
\hline$G_{13}, G_{23}$ & $22.00 \mathrm{GPa}$ & $3.00 \mathrm{GPa}$ \\
\hline$G_{12}$ & $24.00 \mathrm{GPa}$ & $19.5 \mathrm{GPa}$ \\
\hline$\gamma_{13}, \gamma_{23}$ & 0.41 & 0.3 \\
\hline$\gamma_{12}$ & 0.3 & 0.05 \\
\hline$\rho$ & $7600 \mathrm{~kg} / \mathrm{cm}^{3}$ & $1480 \mathrm{~kg} / \mathrm{cm}^{3}$ \\
\hline$\varepsilon_{11}^{s}$ & $0.947 \mathrm{e}-8 \mathrm{~F} / \mathrm{m}$ & $\mathrm{NA}$ \\
\hline$\varepsilon_{33}^{s}$ & $0.668 \mathrm{e}-8 \mathrm{~F} / \mathrm{m}$ & $\mathrm{NA}$ \\
\hline$e_{31}$ & $-8.02 \mathrm{C} / \mathrm{m}^{2}$ & $\mathrm{NA}$ \\
\hline$e_{33}$ & $18.31 \mathrm{C} / \mathrm{m}^{2}$ & $\mathrm{NA}$ \\
\hline$e_{15}$ & $12.84 \mathrm{C} / \mathrm{m}^{2}$ & $\mathrm{NA}$ \\
\hline &
\end{tabular}

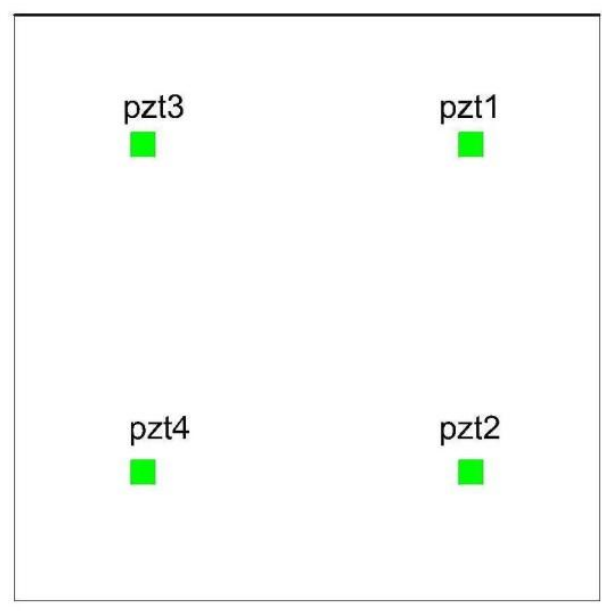

(a)
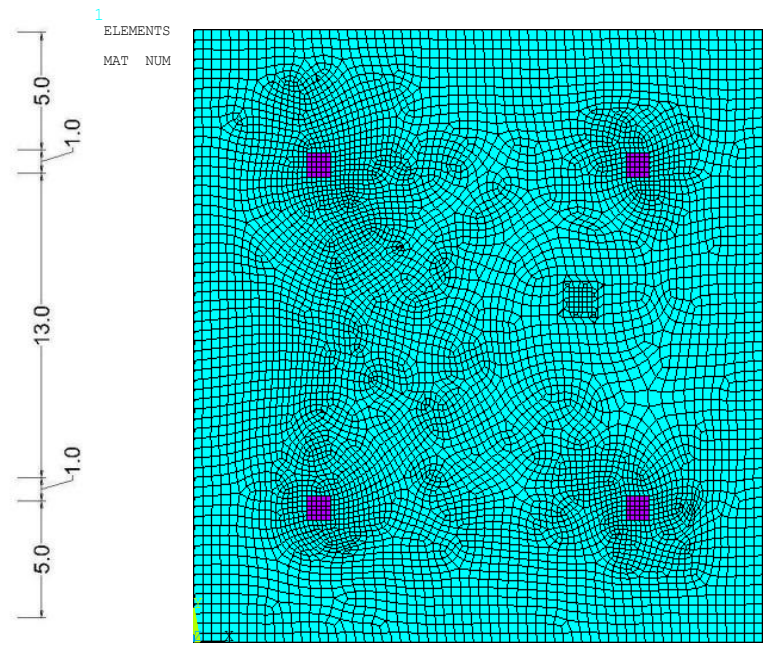

(b)
ANSYS

R15.C

Fig.2. FEM configuration of the composite plate: (a) Geometry in $\mathrm{cm}$ (b) FEM 
In order to model PWAS, SOLID5 element is employed as it has 3-D magnetic, piezoelectric, thermal, and structural field capability with limited coupling between the fields. It is recommended by ANSYS developers for structural and piezoelectric analyses, for its large deflection and stress stiffening capabilities [13]. The element has 8 nodes with up to 6 DOF (thermal, magnetic, and electric DOF in addition to translations in the nodal $\mathrm{x}, \mathrm{y}$, and $\mathrm{z}$ directions). In the current study, four degrees of freedom are considered at each node $\left(\mathrm{U}_{\mathrm{x}}, \mathrm{U}_{\mathrm{y}}, \mathrm{U}_{\mathrm{z}}\right.$, and VOLT DOFs). To achieve convergence, at least 3 to 5 nodes have to be presented per half wave length [14], where

$\lambda=\sqrt{\frac{E}{\rho\left(1-v^{2}\right)}} / f$

Where $\lambda$ is the wave length and $f$ is the frequency .

So, the maximum element size used was $4 \mathrm{~mm}$ to sustain FEM convergence.

Using Rayleigh damping, where

$[C]=\alpha[M]+\beta[K]$

Stiffness matrix multiplier constant $\beta$ is assumed to be $\left(1 \times 10^{-8}\right)$ while mass matrix multiplier constant $\alpha$ is assumed to be zero. Gresil et al [8] demonstrated that the effect of bonding layer doesn't arise under $0.6 \mathrm{MHz}$, consequently, for simplification, the bonding layer was neglected since the frequency range in current study didn't exceed $0.3 \mathrm{MHz}$.

\section{Modal analysis}

The impedance technique is vibration -based technique where

$\mathrm{Z}_{\mathrm{s}}=\frac{\mathrm{F}}{\dot{\mathrm{U}}}$

Where $\mathrm{F}$ is force and $\dot{U}$ is the nodal velocity. The mechanical impedance of host structure is a type of Frequency Response Functions (FRF). FRF is influenced directly by physical characteristics (stiffness, mass and damping) of the structure. The modal analysis was performed through numerical simulation using ANSYS v.15.0 from which the frequency ranges were selected to perform harmonic analysis. The modal analysis was carried out in the range of 0 to $1 \mathrm{MHz}$, in order to cover the adequate range of frequencies employed in the harmonic analysis. The specified frequency range contained 15032 mode shapes. The classification of the frequency ranges was carried out based on the mode shapes characteristics. Sample of the mode shapes configurations are illustrated in Fig.3. The frequency ranges are selected as follows:

- The first range is from 0 to $14 \mathrm{KHz}$ in which most of the modes are separated.

- The second range is from 14 to $58 \mathrm{KHz}$, where the majority of the modes are mixed pattern with flexural configurations.

- The third range is from 58 to $110 \mathrm{KHz}$, as it can be described as closely spaced modes with mixed shear and flexural configurations.

- The fourth range is from 110 to $250 \mathrm{KHz}$, as most of the modes have significant shear configurations.

- The majority of the modes in the range from 250 to $345 \mathrm{KHz}$, exhibited sharp local modes. Also, no significant modes were noticed in the frequency above $345 \mathrm{KHz}$.

Based on the aforementioned characteristics, the first four frequency ranges were employed in the SHM using harmonic analysis. 


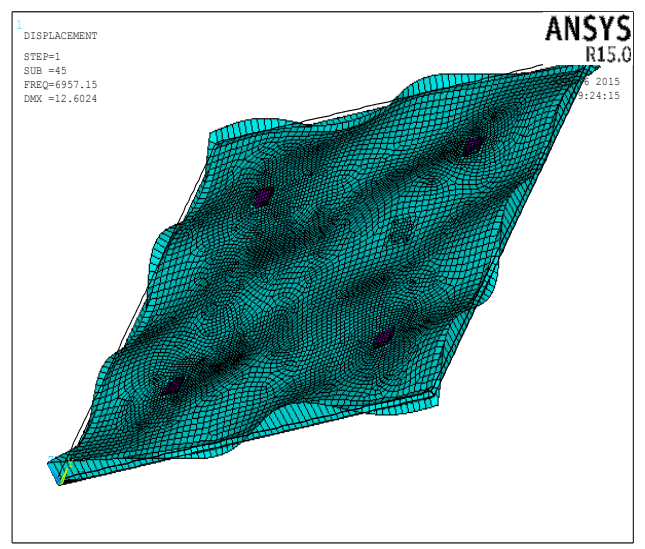

(a)

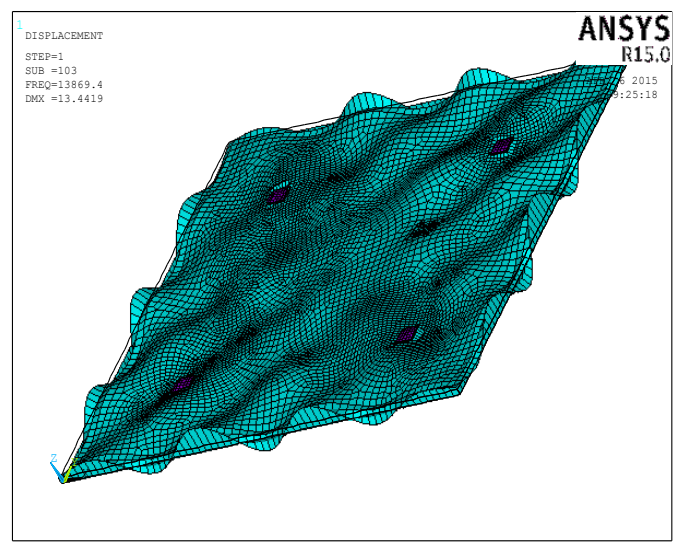

(b)

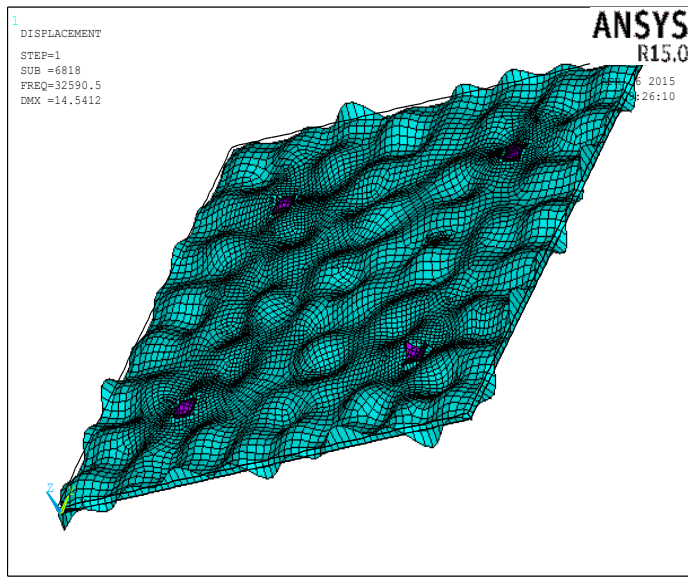

(c)

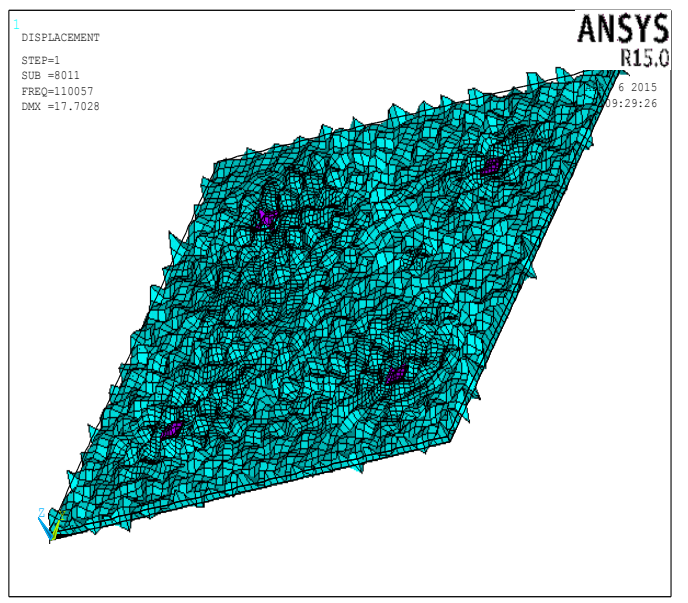

(e)

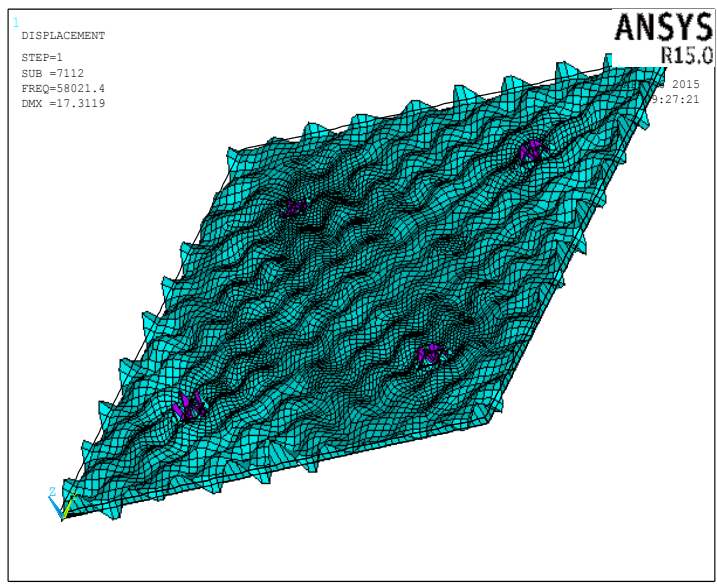

(d)

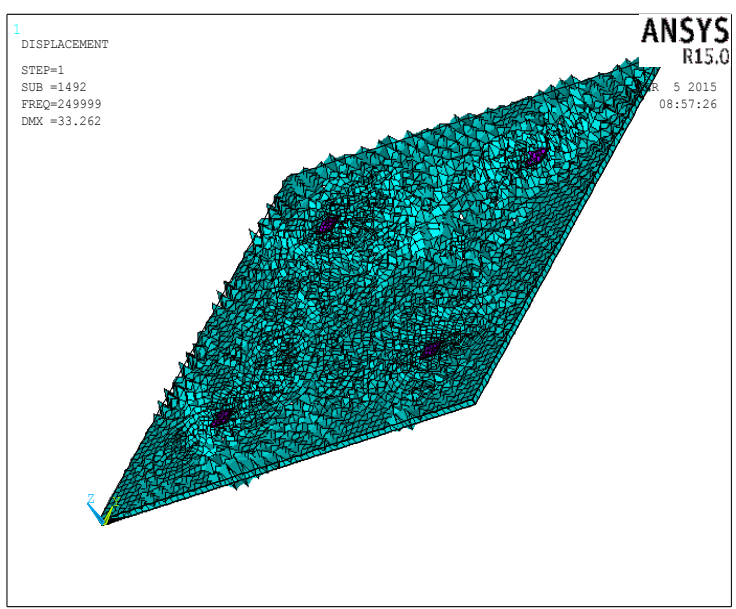

(f) 


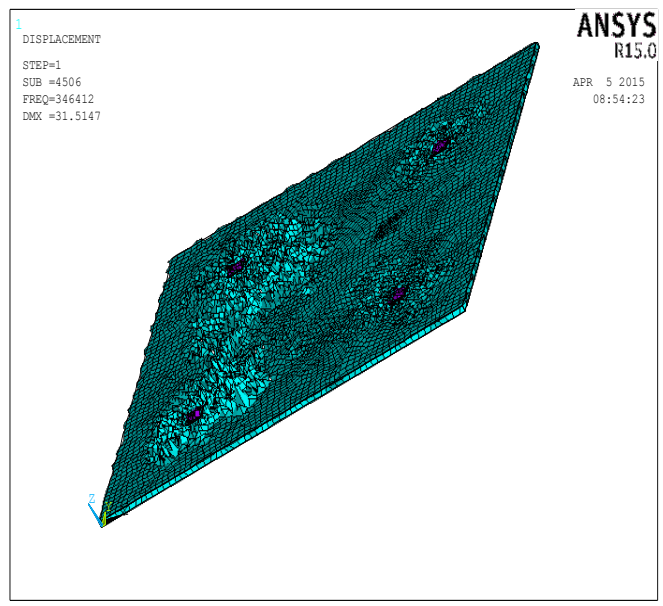

(g)

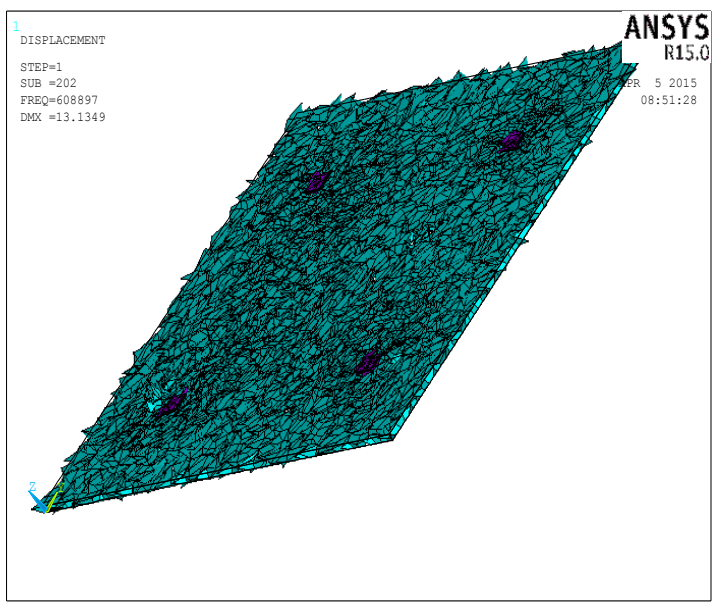

(h)

Fig.3. Sample of Mode shapes at different frequency ranges of interest at frequencies of: (a) $6.9 \mathrm{KHz}$. (b) 13.9 KHz. (c) 32.6 KHz. (d) 58 KHz. (e) 110 KHz. (f) 250 KHz. (g) 346.4 KHz. (h) 608.9 KHz.

\section{Harmonic analysis}

Harmonic analysis was carried out following single- input single-output procedure in which each PWAS was excited at a time over each frequency range to get the EMI spectrum signature of the healthy structure. Equation of motion for piezoelectric coupled field[13] is:

$\left[\begin{array}{cc}{[\mathrm{M}]} & {[0]} \\ {[0]} & {[0]}\end{array}\right]\left\{\begin{array}{l}\ddot{\mathrm{u}}\} \\ \ddot{\mathrm{v}}\}\end{array}\right\}+\left[\begin{array}{cc}{[\mathrm{C}]} & {[0]} \\ {[0]} & -\left[C^{v h}\right]\end{array}\right]\left\{\begin{array}{l}\{\dot{\mathrm{u}}\} \\ \dot{\mathrm{v}}\}\end{array}\right\}+\left[\begin{array}{cc}{[\mathrm{K}]} & {\left[\mathrm{K}^{\mathrm{Z}}\right]} \\ {\left[\mathrm{K}^{\mathrm{Z}}\right]^{\mathrm{T}}} & {\left[\mathrm{K}^{\mathrm{d}}\right]}\end{array}\right]\left\{\begin{array}{c}\{\mathrm{u}\} \\ \{\mathrm{v}\}\end{array}\right\}=\left\{\begin{array}{c}\{\mathrm{F}\} \\ \{\mathrm{L}\}\end{array}\right\}$

Where

$$
\left[C^{v h}\right]=-\frac{1}{\omega^{2}}\left[K^{v}\right]
$$

The above system of equations is simplified to yield the following equations in harmonic analysis as;

$$
\begin{aligned}
& \left(-\omega^{2}[\mathrm{M}]+\mathrm{i} \omega[\mathrm{C}]+[\mathrm{K}]\right)\left(\left\{\mathrm{u}_{1}\right\}+\mathrm{i}\left\{\mathrm{u}_{2}\right\}\right)+\left[\mathrm{K}^{\mathrm{z}}\right]\left(\left\{\mathrm{v}_{1}\right\}+\mathrm{i}\left\{\mathrm{v}_{2}\right\}\right)=\left(\left\{\mathrm{F}_{1}\right\}+\mathrm{i}\left\{\mathrm{F}_{2}\right\}\right) \\
& {\left[\mathrm{K}^{\mathrm{z}}\right]^{\mathrm{T}}\left(\left\{\mathrm{u}_{1}\right\}+\mathrm{i}\left\{\mathrm{u}_{2}\right\}\right)+\left(\left[\mathrm{K}^{\mathrm{d}}\right]-i \omega\left[C^{v h}\right]\right)\left(\left\{\mathrm{v}_{1}\right\}+\mathrm{i}\left\{\mathrm{v}_{2}\right\}\right)=\left(\left\{\mathrm{L}_{1}\right\}+\mathrm{i}\left\{\mathrm{L}_{2}\right\}\right)}
\end{aligned}
$$

Where $\left\{\mathrm{u}_{1}\right\},\left\{\mathrm{v}_{1}\right\}=\{\mathrm{u} \cos \phi\},\{\mathrm{v} \cos \phi\} \quad\left\{\mathrm{u}_{2}\right\},\left\{\mathrm{v}_{2}\right\}=\{\mathrm{u} \sin \phi\},\{\mathrm{v} \sin \phi\}$

$$
\left\{\mathrm{F}_{1}\right\},\left\{\mathrm{L}_{1}\right\}=\{\mathrm{F} \cos \psi\},\{\mathrm{L} \cos \psi\} \quad\left\{\mathrm{F}_{2}\right\},\left\{\mathrm{L}_{2}\right\}=\{\mathrm{F} \sin \psi\},\{\mathrm{L} \sin \psi\}
$$

Hence, the resultant charge $\{\mathrm{L}\}$ on the electrodes are used to calculate the complex current $\overline{\mathrm{I}}=i \omega \sum \mathrm{L}_{\mathrm{i}}$, where $\sum \mathrm{L}_{\mathrm{i}}$ is the nodal charge summation. Complex admittance $\overline{\mathrm{Y}}=\overline{\mathrm{I}} / \overline{\mathrm{V}}$ can easily be obtained knowing input voltage (assigned 1 volt in the current study). Finally get complex EMI $\bar{Z}(\omega)=1 / \bar{Y}$. It was found that the real part of $\bar{Z}(\omega)$ is a good representative for the changes in the modal characteristics of the structure. Harmonic analysis procedure was implemented through numerical simulation using ANSYS v.15.0 for the healthy plate while the EMI spectrum for each PWAS is obtained as a datum. The peaks of the spectrum for the four PWAS array are coincident at the same frequencies with different amplitudes. This can be attributed to the single-input single-output technique followed in the current research. The drawback of this technique is that it doesn't fully excite the some of the mode shapes to its resonance frequency as shown in Fig. 4 which illustrates the EMI spectrum of PZT\#2 and PZT\#4 over two frequency ranges. 


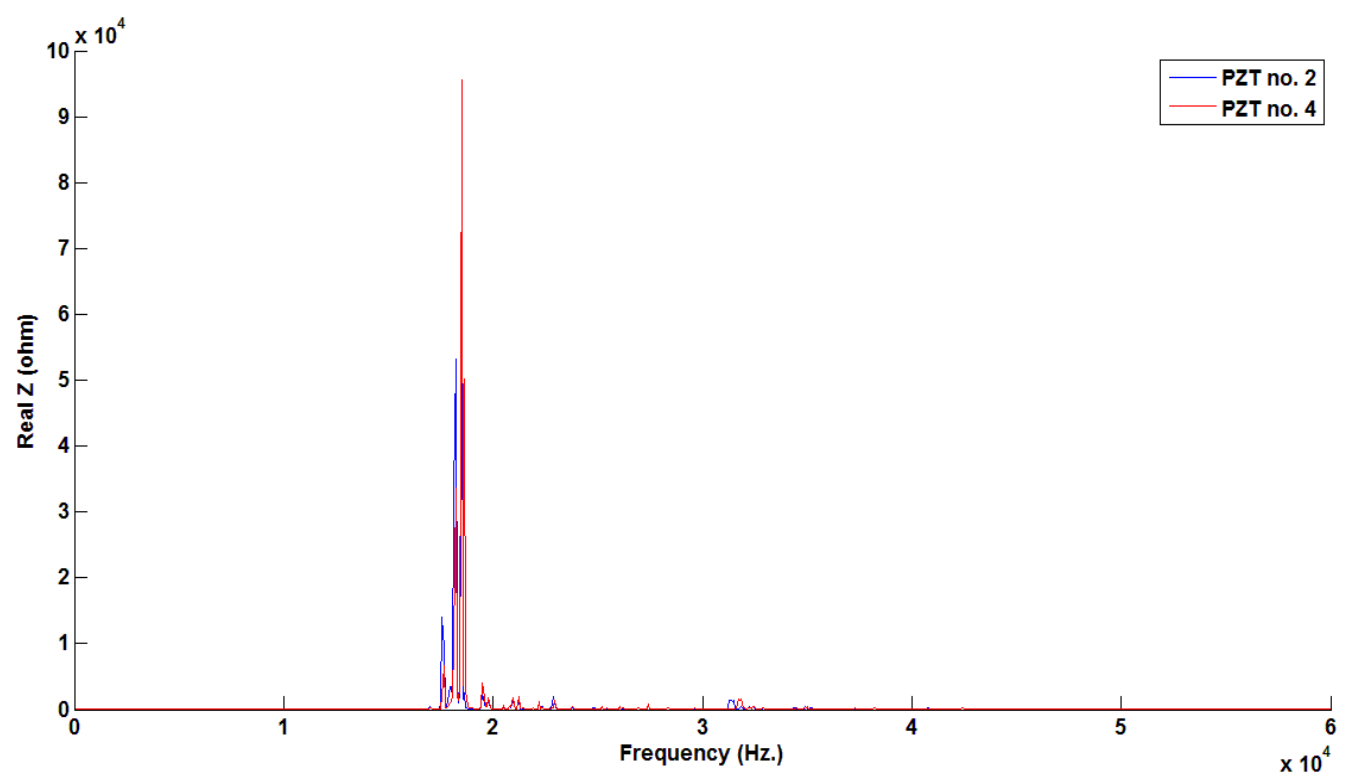

Fig4. (a) : Impedance signature for healthy plate taken by PZT \# 2 vs. PZT no.\# 4 over frequency ranges 0-60 KHz.

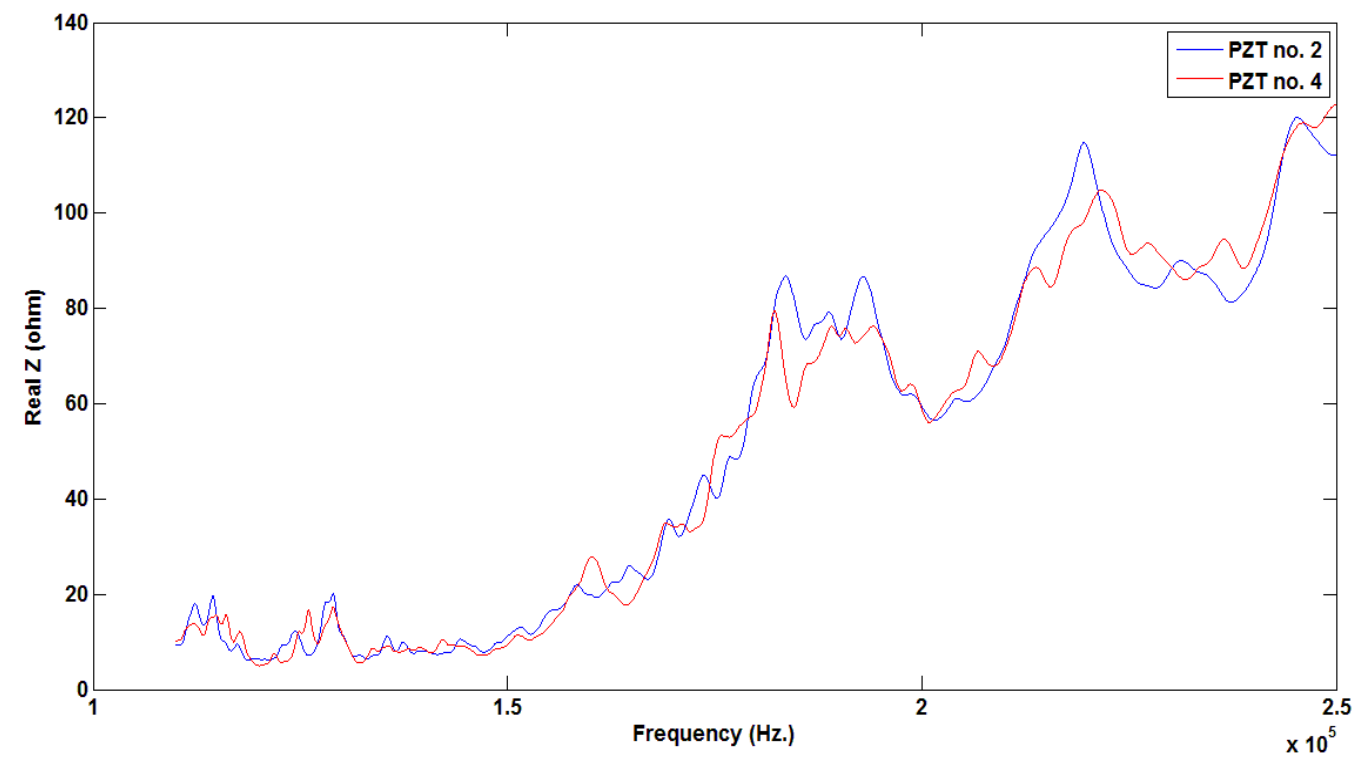

Fig4. (b): Impedance signature for healthy plate taken by PZT \# 2 vs. PZT no.\# 4 over frequency ranges 110-250 KHz.

\section{Simulated Damage Scenarios}

Three damage scenarios were considered during current study represented by reduction of stiffness matrix of an area equal to $10 \times 10 \mathrm{~mm}$ located as shown in Fig. 5. The first damage scenario, classified as (LIGHT) damage, was introduced through $95 \%$ reduction of stiffness matrix of the first lamina of the assigned area. The second damage scenario, classified as (MEDIUM) damage, was introduced through $95 \%$ reduction of stiffness matrix of the first two lamina of the assigned area. Finally, a (SEVERE) damage scenario is simulated by reducing the stiffness matrix of the assigned area for the first four lamina of the same assigned surface area and same stiffness degradation percentage was introduced. The analysis procedure carried out for the healthy plate was repeated for each case of the simulated damage scenarios and the EMI spectrum of defected plate for each PWAS was compared to the spectrum of its healthy counterpart. 


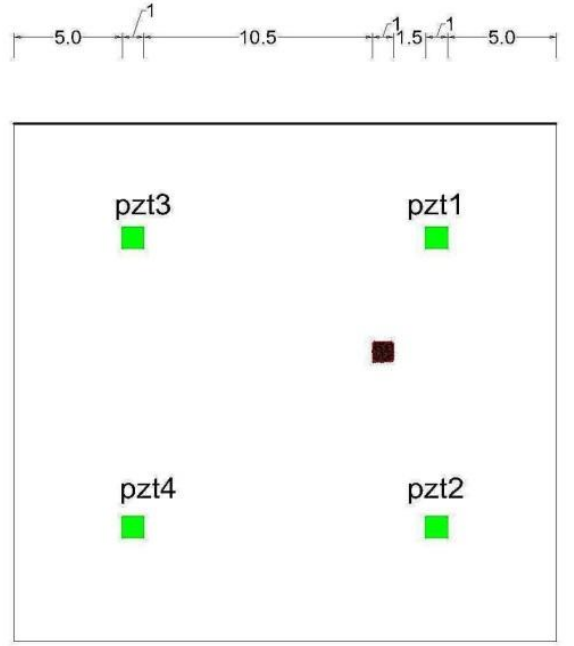

(a)

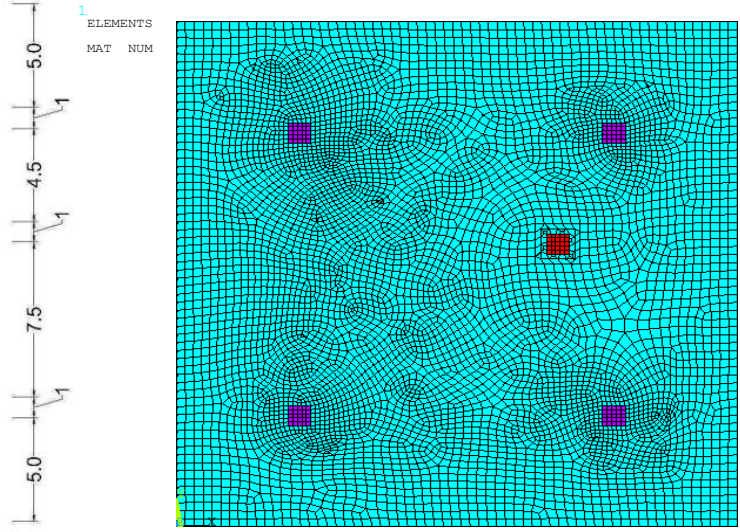

ANSYS

(b)

Fig.5. Configuration of the defected specimen: (a) Geometry in cm (b) FEM

\section{Damage Identification and Results Discussion}

Many statistical tools could be considered as a measure of the differences between healthy and defected states of structures, most familiar one is root mean square deviation (RMSD) which is presented by the formula

$\operatorname{RMSD}=\sqrt{\frac{\sum\left[\operatorname{Re}\left(\mathrm{Z}_{\mathrm{i}}\right)-\operatorname{Re}\left(\mathrm{Z}_{\mathrm{i}}^{\mathrm{o}}\right)\right]^{2}}{\sum\left[\operatorname{Re}\left(\mathrm{Z}_{\mathrm{i}}^{0}\right)\right]^{2}}}$

Where $\operatorname{Re}\left(Z_{i}^{0}\right) \& \operatorname{Re}\left(Z_{i}\right)$ are the real part of EMI of the healthy and defected structure at frequency (i) respectively.

Another statistical tool called damage detection index (DDI) which represented by Napolitano[15] that is defined as

$\mathrm{DDI}=\left(\frac{\sum\left|\mathrm{FI}_{\mathrm{i}}-\mathrm{FD}_{\mathrm{i}}\right|}{\sum \mathrm{FI}_{\mathrm{i}}}\right) \times 100$

Where $\left(\mathrm{FI}_{\mathrm{i}}\right) \&\left(\mathrm{FD}_{\mathrm{i}}\right)$ are the $\mathrm{FRF}$ of the healthy and defected structure at frequency (i) respectively.

Both RMSD and DDI were used to detect the variance in the results of damaged cases from the healthy case. The results were summarized in Table 2.

Table 2. RMSD and DDI results for simulated damage scenario for each frequency ranges

\begin{tabular}{|c|c|c|c|c|c|c|c|c|c|}
\hline \multicolumn{2}{|c|}{$\begin{array}{c}\text { FREQUENCY } \\
\text { RANGE }\end{array}$} & \multicolumn{2}{|c|}{ 0-14 KHz } & \multicolumn{2}{|c|}{ 14-58 KHz } & \multicolumn{2}{|c|}{ 58-110 KHz } & \multicolumn{2}{|c|}{ 110-250 KHz } \\
\hline $\begin{array}{l}\text { PZT } \\
\text { NO. }\end{array}$ & DAMAGE & RMSD & $\begin{array}{l}\text { DDI } \\
\%\end{array}$ & RMSD & $\begin{array}{l}\text { DDI } \\
\%\end{array}$ & RMSD & $\begin{array}{l}\text { DDI } \\
\%\end{array}$ & RMSD & $\begin{array}{l}\text { DDI } \\
\%\end{array}$ \\
\hline \multirow{3}{*}{ PZT1 } & LIGHT & 0.4070 & 29.48 & 0.4388 & 26.51 & 0.0047 & 0.69 & 0.0092 & 0.72 \\
\hline & MEDIUM & 0.5261 & 34.18 & 0.8512 & 49.00 & 0.0102 & 1.52 & 0.0131 & 1.07 \\
\hline & SEVERE & 1.1458 & 45.20 & 0.8739 & 56.77 & 0.0181 & 3.00 & 0.0169 & 1.49 \\
\hline \multirow{3}{*}{ PZT2 } & LIGHT & 0.5362 & 28.35 & 0.2630 & 19.14 & 0.0064 & 0.75 & 0.0061 & 0.51 \\
\hline & MEDIUM & 2.8077 & 60.41 & 0.4014 & 32.14 & 0.0148 & 1.75 & 0.0112 & 0.94 \\
\hline & SEVERE & 3.1662 & 70.98 & 0.4527 & 41.83 & 0.0289 & 3.54 & 0.0143 & 1.27 \\
\hline \multirow{3}{*}{ PZT3 } & LIGHT & 0.3817 & 23.62 & 0.2897 & 22.81 & 0.0030 & 0.49 & 0.0036 & 0.29 \\
\hline & MEDIUM & 0.3840 & 24.84 & 0.4829 & 38.63 & 0.0071 & 1.14 & 0.0073 & 0.61 \\
\hline & SEVERE & 1.1923 & 43.73 & 0.6364 & 54.01 & 0.0159 & 2.43 & 0.0099 & 0.79 \\
\hline \multirow{3}{*}{ PZT4 } & LIGHT & 0.4758 & 28.60 & 0.5414 & 23.29 & 0.0027 & 0.47 & 0.0021 & 0.17 \\
\hline & MEDIUM & 0.5521 & 33.01 & 1.248 & 50.63 & 0.0059 & 1.04 & 0.0043 & 0.35 \\
\hline & SEVERE & 0.8574 & 45.11 & 1.3476 & 63.23 & 0.0142 & 2.26 & 0.0076 & 0.62 \\
\hline
\end{tabular}


The values of the indices listed in Table 2., for each damaged scenario, over different frequency ranges illustrate the relationship between the index value and the damage severity. The non-zero values for the damaged indices can be considered as a reliable measure for damage propagation. Both indices increase as the severity of the damage does for the same PWAS over the same frequency range as shown in Fig.6. Examining the indices values led to the conclusion that: indices values are too small in the higher frequency ranges compared to the indices in the lower frequency ranges for the same damage scenario and the same PWAS as shown in Fig.7. This conclusion can be easily reached by examining the mode shapes in each frequency range of the host structure. The closer PWAS to the damage place the higher indices values for all damaged scenarios specifically in the higher frequency range as shown in Fig.8. This can be attributed to the reason that the modes that contain valuable modal data are in the higher frequency ranges.

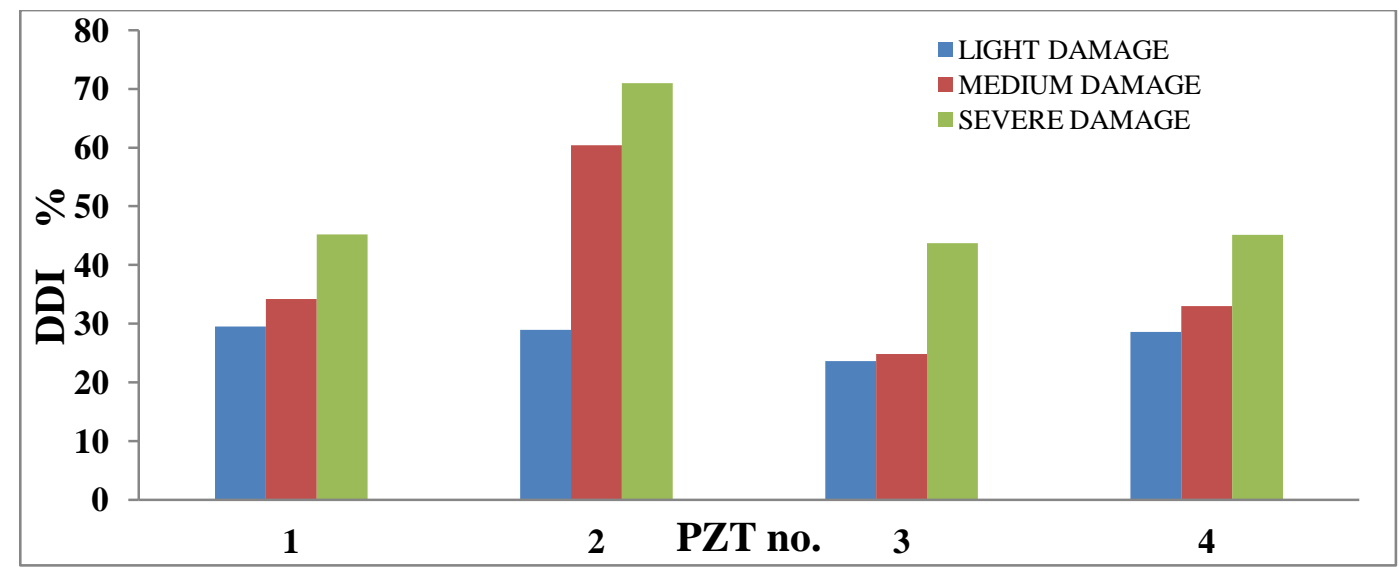

(a)

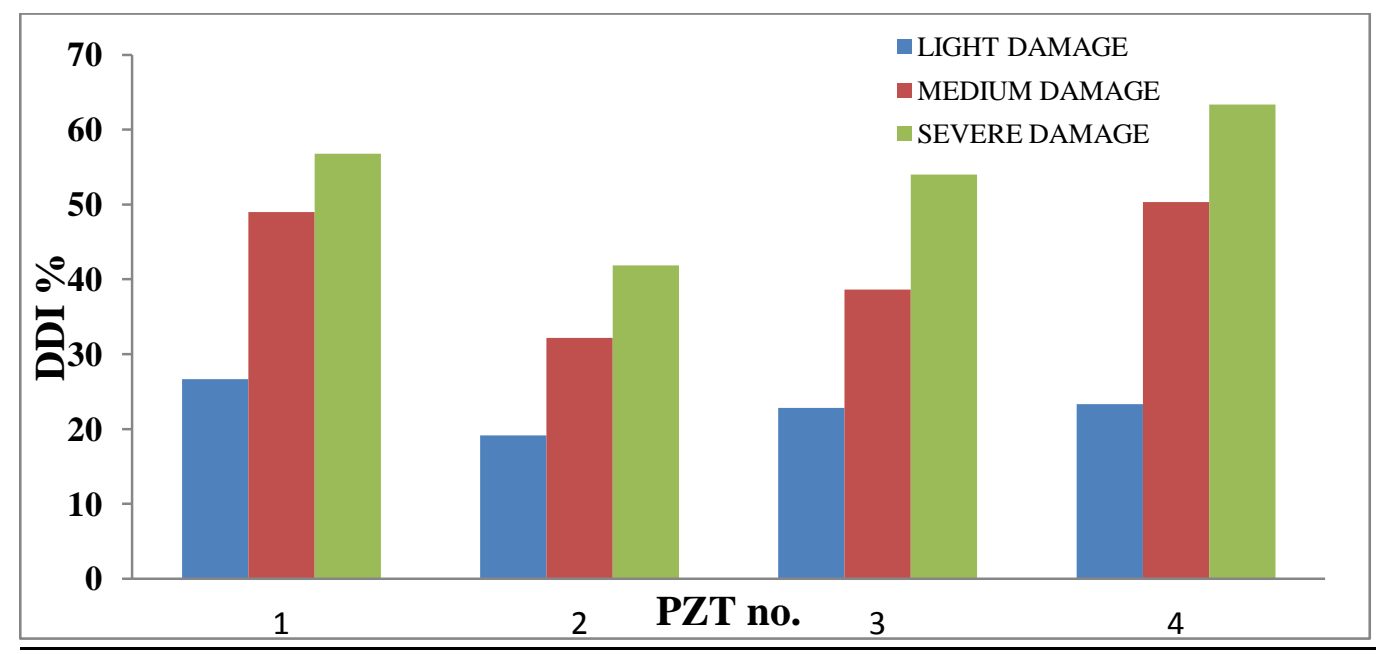

(b)

Fig.6. Damage detection indix (DDI) for each PZT with different damage severity over: (a) 0-14 KHz (b) 14-58 KHZ. 


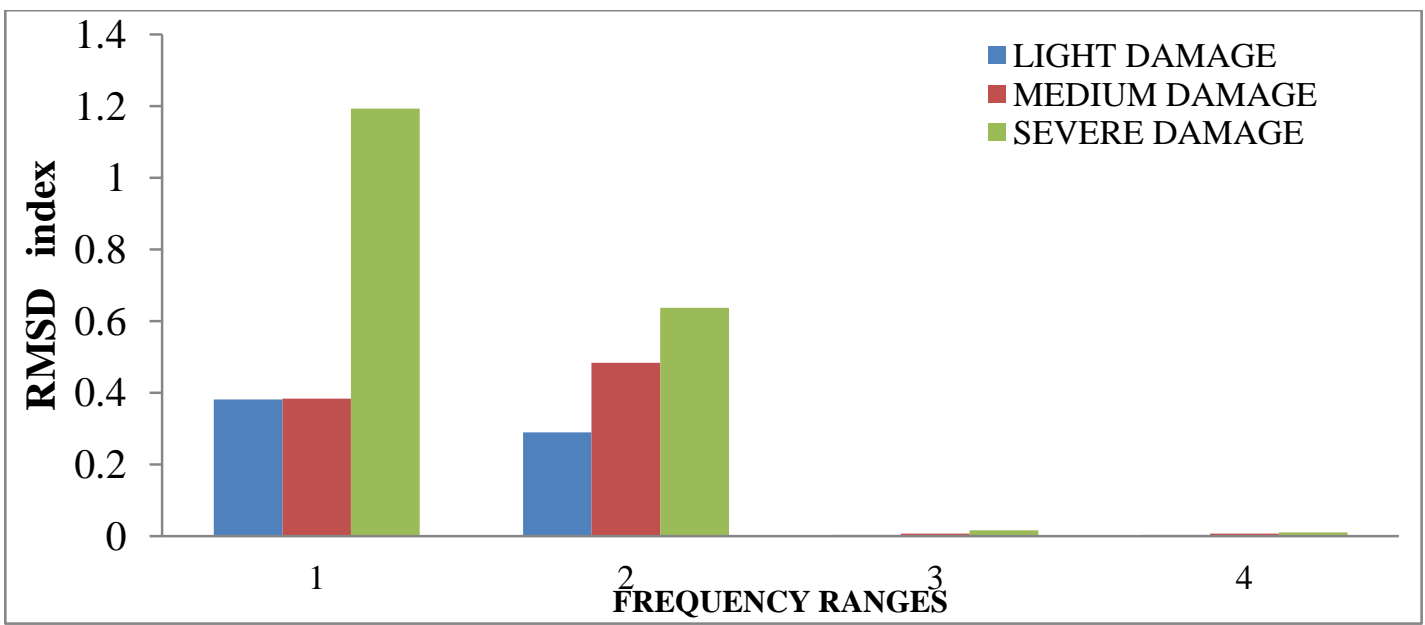

Fig.7. RMSD index for PZT no.3 over the 4 frequency ranges considered in the study with different damage cases.

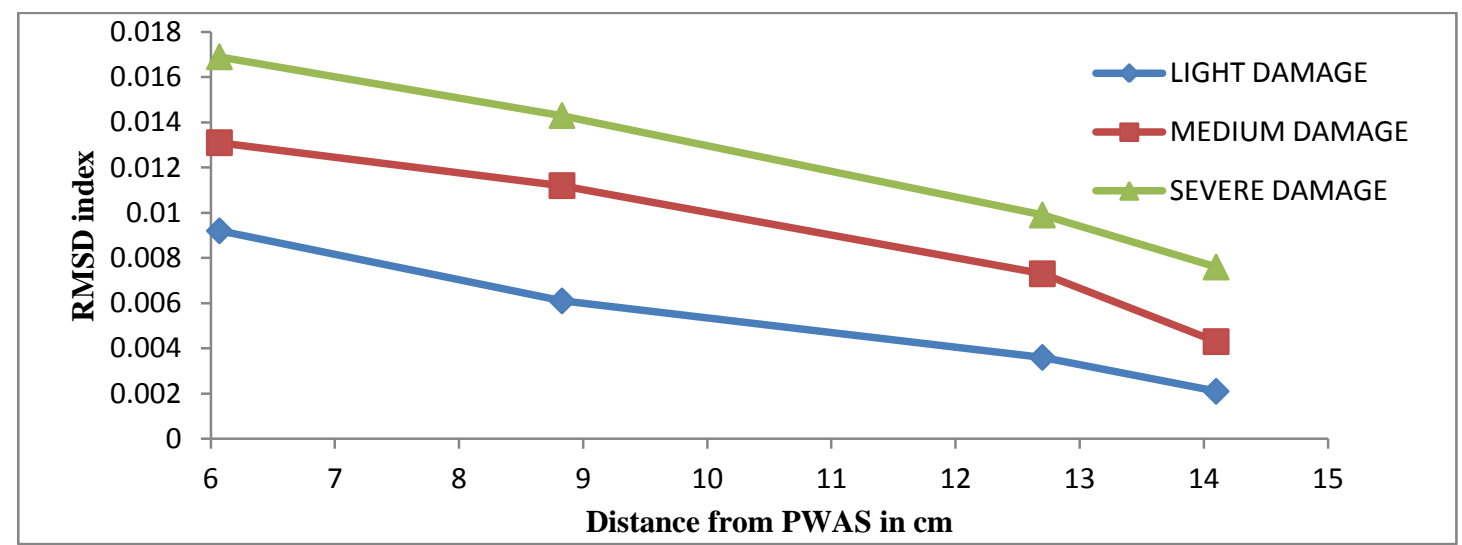

(a)

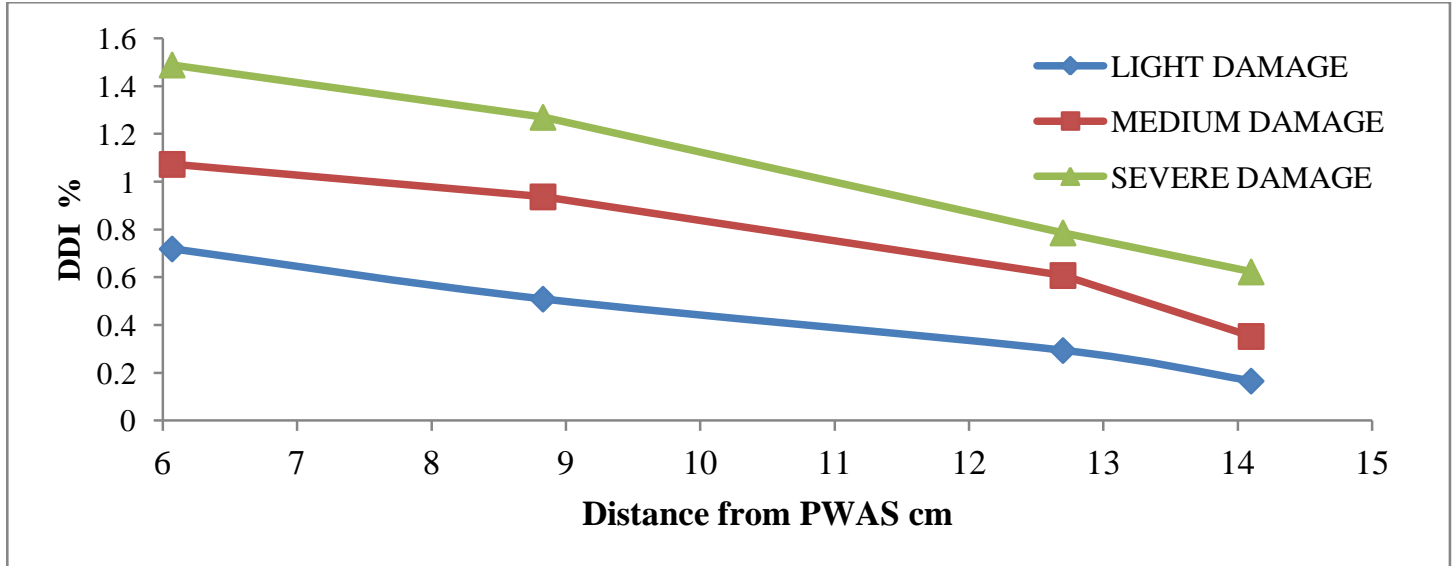

(b)

Fig.8. Relation between distance from damage to each PZT and (a) RMSD index (b) DDI over frequency range 110-250 KHz.

\section{Conclusion}

The implementation of finite element model, represented by multi-physics ANSYS software, found to be beneficial to model the proposed SHM system using EMI technique. This is more efficient, especially when it is used for sophisticated non-homogenous or anisotropic materials which are considered extraordinary challenges for the other limited analytical approaches. However, there are some difficulties facing such model among them are: the necessity of large data storage memory due to the need for fine meshing in order to achieve 
convergence. Also, the need for FE model updating procedure to consider the dielectric and mechanical losses from experimental measurements for the healthy structure at early stage of SHM system design.

Noticeable fluctuation between the amplitudes of resonance for the healthy structure measured by different PWASs, especially at low frequencies, can be attributed to single-input single-output procedure followed in the simulated modal analysis. These procedures do not allow all modes to be fully excited.

Three damage scenarios were introduced (Light, medium and sever). The effect of the degradation in stiffness is reflected in the measured statistical indices, RMSD and DDI. The indices are calculated from the difference between healthy and damaged plate spectrums. It is found that RMSD and DDI can be effectively used to localize damage and its propagation through the laminas. The indices values found to function of the damaged severity. Also, for the same damage scenario, the indices values are proportional to the distance between the PAWS and damage location. The SHM algorithm introduced in the current research found to be relatively successful in identifying damage in plate made of composite material for different damage levels. However, to establish promising, effective, and robust SHM system, a more reliable mathematical methodology need to be employed for the implementation of the algorithm in larger plate or structure such as Artificial Neural Network (ANN).

\section{References}

1. G.L. anderson, A.C.J.C., Introuduction to smart structures, in Inellegent structural systems, H.S.T.L. Anderson, Editor. 1992, Kluwer Academic Publishers: Netherlands.

2. Farrar, G.P.a.C.R., Piezoelectric impedance methods for damage detection and sensor validation in Encyclopedia of structural health monitriong, F.-K.C.Y.F. Christian Boller, Editor. 2009, John Wiley \& Sons, Ltd. p. 365-377.

3. C. LIANG, F.P.S., C. A. ROGERS, Coupled Electro-Mechanical Analysis of Adaptive Material systems - Determination of the Actuator Power Consumptionand System Energy Transfer. Journal of Intelligent Material Systems and Structures, 1994. Vol. 5: p. 1220 .

4. Zhou, S., C. Liang, C.A. Rogers, A Dynamic Model of Piezoelectric Actuator-Driven Thin Plates,, in SPIE Smart Structures and Materials Conference. 1996: Orlando, Florida. p. 550-562.

5. Victor Giurgiutiu, A.N.Z., Characterization of Piezoelectric Wafer Active Sensors. JOURNAL OF INTELLIGENT MATERIAL SYSTEMS AND STRUCTURES, 2000. Vol. 11: p. pp.959-975

6. Ikeda, T., Fundamentals of Piezoelectricity. 1996: Oxford university press.

7. Bhalla, S., A Mechanical Impedance Approach for Structural Identification, Health Monitoring and Non-Destructive Evaluation Using Piezo-Impedance Transducers., in Civil and Enviromental Engineering. 2004, Nanyang Technological University.

8. Matthieu Gresil, L.Y., Victor Giurgiutitu, Micheal Sutton, Predictive modeling of electromechanical impedance spectroscopy for composite materials. Structural Health Monitoring, 2012.

9. Pierre Selva, O.C., Valerie Budinger, Frederic Lachaud, and Joseph Morlier, Smart monitoring of aeronautical composites plates based on electromechanical impedance measurements and artificial neural networks. Open Archive Toulouse Archive Ouverte, 2013. 56: p. 794-804.

10. F., L., Modelling of induced strain actuation of shell structures. 1995, Virginia Polytechnic Institution and State University: Blacksburg. 
11. Lim YY, B.S.a.S.C., Structural identification and damage diagnosis using self-sensing piezo-impedance transducers. Smart Materials and Structures, 2006. 15(4): p. 987-995.

12. Physical and Piezoelectric Properties of APC Materials.

13. Mechanical APDL Release 0.15, in ANSYS, Inc Products 2013.

14. Makkonen, T., Holappa, A., J., and salomaa, M., Finite Element SImulation of TinFilm Composite BAW Resonators, in IEEE Transactions on Ultrasonics, Ferroelctrics and Frequency Controrl. 2001. p. 1241-1258.

15. Napolitano, L., Fedele, P., Viscardi, M. and Lecce, L., Damage Identification and Location on Typical Aeronautical Structure. SPIE 1998. 3397. 